\title{
A Study on the Factors that Influence Wearable Users' Quantified Self Based on UTAUT Model
}

\author{
Hong Jin ${ }^{1}$, Jiayue Yan ${ }^{2}$ \\ ${ }^{1,2}$ School of Business, Jiangxi normal university, China
}

\begin{abstract}
Background/Objectives: Based on the existing user acceptance model, this paper proposes a quantitative selfacceptance model for wearable device users, and puts forward relevant assumptions and provide advice on the development of wearable technology. Methods/Statistical analysis: In this paper, we set the hypothesis based on the results of previous studies. This was structured and a questionnaire survey was conducted on users on a Packard 5-point scale through a questionnaire. Analytical tools are SPSS and AMOS. Findings: In terms of theoretical research, the model proposed in this study is limited by the degree of development of the research object, and the variables and interrelationships of the model are relatively simple. According to the previous research experience, the model still has a lot of room for improvement, and it has yet to be optimized in terms of complexity and effectiveness. Improvements/Applications: All in all, this research has achieved the initial research purpose, completed the research task of the factors affecting the willingness of wearable device users to accept, has some innovation in the academic theory level, and has certain reference significance for practical activities.
\end{abstract}

\section{Index Terms}

Distributed Network, Machine Learning, Block-chain, Load-Balancing, Performance Improvement

\footnotetext{
Corresponding author : Hong Jin

com342944219@qq.com

- Manuscript received April 20, 2020.

- Revised May 8, 2020 ; Accepted June 10, 2020.

- Date of publication June 30, 2020.

(C) The Academic Society of Convergence Science Inc.

2546-1583 @ 2017 IJEMR. Personal use is permitted, but republication/redistribution requires IJEMR permission
} 


\section{INTRODUCTION}

In recent years, artificial intelligence has become a hot word in society, and artificial intelligence products have gradually entered into people's lives. Users continue to participate in quantitative selfactivity, will also promote the development of smart wearable technology.

At present, there is a lack of quantitative research on both wearable computing and quantified self. It is just speculation based on theory. The factors that affect the quantified self of wearable device users lack certain analysis.

The purpose of this study is to use the user acceptance model to put forward the influencing factors of users' acceptance of wearable devices to quantify themselves, and to propose the development of the quantitative market for wearable devices from a theoretical level.

\section{LITERATURE REVIEW}

\section{A. Overview of the development of wearable devices}

The development of wearable devices in the information technology market was first initiated by Google Glass, which was released by Google.

As a wearable smart device, coupled with the improvement of artificial intelligence technology, more businesses see business opportunities. Wearable devices have further weakened the traditional machine concept, allowing portable ways to integrate wearable devices into users' lives [1].

At present, wearable devices on the market are mainly concentrated in three categories: smart bracelets, smart watches and smart glasses, but their essential characteristics determine that wearable devices still have a lot of room for development and application market.

As users 'awareness of quantifying themselves gradually increases, wearable devices will provide users with more personalized development solutions and high-quality service experience through the collection and intelligent analysis of users' own data.

\section{B. User acceptance theory}

The quantified self enters our lives silently. The research of quantifying self is mainly concentrated in the field of medical health. Few studies have analyzed the influencing factors of quantified self from the perspective of users' wearable devices.

Quantified self refers to the process of self-tracking of the individual's own physiology, behavior or surrounding environment information,real-time measurement and recording of individual life data and self-adjustment based on data feedback [2].

With the rapid development of artificial intelligence and wearable technology, quantifying the self and the increasingly popular smart wearable devices make it easier and easier for individuals to collect their own data, observe their physical conditions, and deepen their self-knowledge.

Users can monitor self at any time with the help of wearable devices and other quantitative tools to accurately implement behavior choices. Enterprises conduct product development and design, customer segmentation and precise marketing based on user self-quantitative data to objectively predict and drive expected consumer behavior [3].

\section{C. quantified self}

Wearable technology is an innovative information technology product. The user's willingness to participate is in line with the user technology theory research. The focus of the user acceptance theory is on the user's perception of the value of information technology.

The level of value is the core of the current user acceptance theory research. User acceptance theory has also undergone evolution and exploration at different stages. The first stage is based on the acceptance study of the rational behavior theory.

The representative theories proposed are the rational behavior theory TRA [4] and the planned behavior theory TPB [5]; The second stage is based on the user's acceptance of the technology itself, represented by the technology acceptance models TAM and TAM2; the third stage is Venkatesh [6] based on the technology update and development combined with the above technology acceptance model to propose a more complete technology Accept and use the integrated model UTAUT.

The model believes that the user's performance expectations, effort expectations, community influence and convenience conditions will affect the user's willingness to use, and then impress the user's use behavior. At the same time, the four variables are regulated by age, experience, use experience, and voluntary use[7-9].

\section{RESEARCH MODELS AND ANALYSIS}

In summary, this article will redefine the definition of variables in the original model for the particularity of wearable devices[10,11].

The model is more suitable for wearable device user acceptance models. At present, there is still a lack of research on related factors of wearable device users.

Although the UTAUT model has certain universality, the path from wearable device users' quantification of self-will to participation behavior is still difficult to fully support, and it is impossible to complete this relationship The integrity is verified. 
Therefore, this study only studies the quantitative self-influencing factors of wearable users. The model structure is shown in Figure 1.

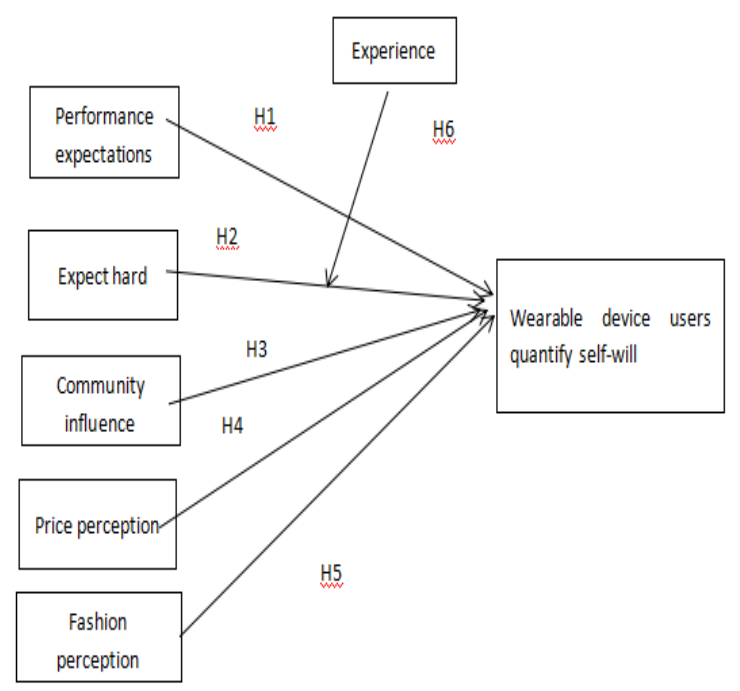

Fig. 1. Model of factors affecting the quantified self-will of wearable device users

\section{H1: Performance expectations encourage users to accept wearable devices to quantify themselves.}

\section{H2: Efforts are expected to promote users to accept wearable devices to quantify themselves.}

\section{H3: Community influence has a significant role in promoting the acceptance of wearable devices by users.}

\section{H4: Price perception has a significant effect on users' acceptance of wearable devices.}

H5: Use experience plays a regulatory role between performance expectations and user acceptance.

(1) Performance expectations refer to users' expectations about the usefulness perception and results of wearable devices. Users use wearable devices to quantify themselves, which increases their self-knowledge and can bring more convenience to their lives.

Based on the UTAUT model, when users accept a wearable device, they will pay attention to the actual meaning and value of using the device.

(2) The effort expectation corresponds to the userperceived ease of use in the TAM model of technology acceptance theory. The purpose of wearable is to allow customers to rely on wearable technology, so as to habitually participate in quantifying self-activities. Shin [7] believes that when users have a stronger perception of technology acceptability, users' satisfaction is stronger.

Based on the UTAUT model, when accepting a wearable device, the user pays attention to the effort cost of using the wearable device. If the user thinks it is easy to use the wearable device and the technical operation is controllable, then the user is willing to accept the wearable device.

(3) Community influence, that is, the user's social environment's recognition and recognition of wearable devices, and the general views of members of the society on wearable devices and their users.

Assuming that it comes from the UTAUT model, the social environment will have a certain impact on the user's purchasing behavior. The wearable device is also used for decoration and can also be used to show the owner's social impression.

(4) Price perception is the user's perception of the price status and value preservation of wearable devices. Higher price perception has a depressing effect on users' acceptance behavior. This view has been confirmed in user acceptance fields such as $3 \mathrm{G}$ mobile network services at home and abroad.

When a user intends to purchase a wearable device, the product price will be considered. If the user believes that the price of the wearable device is in line with psychological expectations, the price is equivalent to the actual use value, the user accepts.

(5) Use experience: the user's experience of using similar technologies or similar products or solutions to typical technical problems. Since the current series of wearable devices are not widely circulated on the market, you can use the experience of wearable devices to analyze the acceptance of wearable devices.

The user's previous experience is better, then the experience will play a positive role in regulating the relationship between performance expectations and use acceptance, and vice versa.

\section{CONCLUSION}

Although many scholars continue to study in the field of user acceptance willingness and acceptance behavior, the theory in this field has also been greatly enriched, and it has been repeatedly verified in practice, but it is specific to the emerging information technology represented by wearable devices.

At present, there is no relevant research. Therefore, this research starts from the perspective of quantifying self, and explores the main influencing factors of users' quantification of wearable devices.

In terms of theoretical research, the model proposed in this study is limited by the degree of development 
of the research object, and the variables and interrelationships of the model are relatively simple. According to the previous research experience, the model still has a lot of room for improvement, and it has yet to be optimized in terms of complexity and effectiveness.

The integration and interpretation of variables are still to be discussed. In terms of practical application, the conclusions drawn by this study are only the results of theoretical analysis, which can only provide limited reference value for actual business activities, and cannot be used as a solid basis for business behavior.

All in all, this research has achieved the initial research purpose, completed the research task of the factors affecting the willingness of wearable device users to accept, has some innovation in the academic theory level, and has certain reference significance for practical activities. There is nothing in this article that needs scholars to criticize.

\section{Acknowledgments}

This study is supported by the National Natural Science Foundation of China (No.71962014) and Thirteenth Five-Year Planning (2017) research project of Jiangxi Social Science (No.17GL05).

\section{REFERENCES}

[1] Bartlett SJ, Barnes Dong-fang li.(2015). Wearable equipment industrial chain and developing trends of the study. Guangzhou: guang dong province academy of social sciences.

[2] Chen Gen.(2014) Wearable devices, mobile Internet new wave . Beijing: mechanical industry press, 5 .

[3] Chen Ran, Yang cheng.(2014).Quantified self: big data era education field study new opportunities horizon report 2014 enlightenment . Journal of modern education technology, 24 (11) : 5-11.

[4] Zhang yudong, li dongjin.(2008) Research on the obstacle factors and the influencing mechanism of consumer participation in quantifying self . Journal of management, 15 (01) : 74-83.

[5] T, RA Mcivor. Integrating patients into meaningful real-world research. Ann Am Thorac Soc, 2014,11(Suppl 2):S112-117.

[6] Ajzen I, Fishbein M.(1980).Understanding attitudes and predicting social behavior .Preticehall,35-55.

[7] Jin, H., Chi, C., \& Gao, X. (2018, December). Strategic Research on Accurate Marketing to Enhance Consumer Experience of Social Media Users. In 2018 2nd International Conference on Economic Development and Education Management (ICEDEM 2018). Atlantis Press.

[8] Jin, H., Duan, G., \& Park, S. T. A Study on Motivation to Consumer Social Information Search. INTERNATIONAL JOURNAL OF EMERGING MULTIDISCIPLINARY RESEARCH (IJEMR), 4(1), 7-10.

[9] Li, G. (2019). An Introduction to the Mobile Payment Market and Trend in China. INTERNATIONAL JOURNAL OF EMERGING MULTIDISCIPLINARY RESEARCH (IJEMR), 3(1), 1-5.

[10] Jin, H., Wang, H., Park, S. T., \& Kim, Y. K. Research on Marketing Strategy of Traditional Retailers under the Background of Internet-A Case Study of Wal-Mart. INTERNATIONAL JOURNAL OF EMERGING MULTIDISCIPLINARY RESEARCH (IJEMR), 2(3), 27-32.
[11] [11] Jin, H., Miao, Y., \& Park, S. T. (2018). A Case Study of Marks and Spencer lost China. Journal of Industrial Convergence, 16(2), 15-23. 